\title{
Multiple coupling modes to relax shear strain during grain boundary migration
}

\author{
N. Combe $e^{a, b, *}$, F. Mompiou ${ }^{a, b}$, M. Legros ${ }^{a, b}$ \\ ${ }^{a}$ Centre d'Elaboration de Matériaux et d'Etudes Structurales, CNRS UPR 8011, 29 rue J. Marvig, BP 94347, 31055 \\ Toulouse cedex 4, France \\ ${ }^{b}$ Université de Toulouse ; UPS ; F-31055 Toulouse, France
}

\begin{abstract}
Shear-coupled grain boundary (GB) migration is an effective plastic mechanism in absence of dislocation activity, ie. more favorably in nanocrystalline metals. For a given GB, several stress induced migration mechanisms, referred as coupling modes participate to the decrease of the elastic energy produced by the shear. They operate through the nucleation and motion of interfacial defects known as disconnections, carrying elementary shear strain characterized by their Burgers vector. However, so far, the coupling modes have been studied only under a simple shear, a situation much less complex than expected in a strained polycrystal, where multiple components of the stress tensor are present. Here we propose a more systematic investigation of the coupling modes when a composite shear is applied. This promotes the activation of new coupling modes. Using Molecular Dynamics simulations, we evidence these multiple coupling modes and the operation of their associate disconnections. Moreover, we also show that, even at low temperature, GB migration may occur by the successive occurrence of two modes: the relaxed shear appears then as an effective parameter, resulting from the combination of two operating elementary mechanisms.
\end{abstract}

Keywords: Disconnection and dislocation, Grain boundaries, Vacancies, Plastic deformation, Molecular Dynamics

In absence of dislocations activity, grain boundary (GB) migration was found to be an efficient plastic deformation mechanism [1. Among different GB migration mechanisms, shear coupled grain boundary migration (SCGBM) is considered particularly efficient, especially at low temperature, because it is a conservative mechanism, analogous to dislocation slip. For this reason, SCGBM has drawn a significant attention. During SCGBM, the GB migrates over a distance $h$ coincidentally with the relative displacement $b$ of the two grains. The resulting shear, also known as the coupling factor $\beta=\frac{b}{h}$ is commonly used to characterize the mechanism.

In the early seminal work of Cahn et al., a theoretical description of SCGBM was first proposed, based on the motion of intrinsic GB dislocation networks [2, 3, 4]. The nature and density of these dislocations can be formally determined through the Frank-Bilby equation (FBE) [5]. The multiplicity of solutions of the FBE, potentially leads to several coupling modes associated to different coupling factors. Cahn et al. 3 have predicted for instance, in FCC crystals, four coupling modes in symmetric [001]-tilt GBs. While three of them have been evidenced in Molecular dynamics simulations [3, 6, 7, 8, 9], only two modes have been experimentally observed in macroscopic sheared Al bicrystals [10, 11, 12,

More recently, it has been found that the elementary processes of SCGBM are not induced by the motion of the intrinsic dislocations but by the nucleation and motion of disconnections 13, 14. A disconnection is a GB defect with both a step and a dislocation character. Both experiments and simulations have evidenced the role of these disconnections during the SCGBM [15, 16, 17, 18, 13, 19, 8, 9, 20, 21, 22. The coupling factor of the SCGBM is directly related to the ratio of the (norm of the) Burgers vector to the step height of

\footnotetext{
* Corresponding author

Email addresses: combe@cemes.fr (N. Combe), mompiou@cemes.fr (F. Mompiou), legros@cemes.fr (M. Legros)
} 
the mobile disconnection. Hence, a coupling mode is due to the successive nucleation and displacement of a disconnection along its GB plane. Numerous disconnection types can exist for a given GB. In a coincident GB, they can be enumerated: their Burgers vectors correspond to the translations that preserve the GB lattice structure, forming the so-called Displacement Symmetry Conserving (DSC) lattice. Among all these disconnections, those with Burgers vectors parallel to the GB plane can glide and are thus favored during the SCGBM 11. But even with this limitation, the number of potential operating disconnections remains large, while only two modes have been experimentally observed in FCC [001]-tilt GBs [10].

Very few numerical studies have reported coupling factors incompatible with the prediction of the FBE based model. Among them, some simulations are performed at high temperature [23, 24] so that the coupling factor presumably corresponds to an effective coupling factor as proposed recently [22], involving several elementary SCGBM events (and several disconnections). Experimentally, especially in small grained polycrystalline metals, low coupling factors have been measured which can be interpreted as alternative modes but more presumably from a mixture of coupling modes. [17, 25. It should be noted moreover that at variance to ideal bicrystals submitted to a simple shear, GBs in real strained polycrystals are expected to feel locally a more complex stress field. We emphasize that the resolved shear stress on a GB can take any direction and that some plastic mechanisms should operate in order to relax the associated elastic energy. More interesting for our present problematic are two studies 26, 27 that have investigated the SCGBM of a symmetric $\sigma 11(131)-[110]$ GB in four directions some of them involving component along the GB tilt axis and two asymmetric $\sigma 3(\overline{1} \overline{1} 5) /(111)-[\overline{1} 10]$ and $\sigma 9(115) /(111)-[\overline{1} 10]$ GB in eight directions. These studies actually report the operation of several disconnections incompatible with the prediction of the FBE based model and that allows to relax the elastic energy accumulated by the stress.

In this manuscript, we propose to extend these latter studies by investigating a series of GBs under the application of a general shear stress and by systematically examining the disconnections involved in the SCGBM. We show that numerous disconnections and thus modes are available to the system in order to relax the shear elastic energy. We have chosen here to limit our study to shear stress, eluding stresses with components normal to the GB plane that have been investigated elsewhere [9, 28,

Investigating numerous GBs in FCC materials, we focus on homogeneous nucleation of disconnections and we evidence some elementary SCGBM events presumably incompatible with the prediction of the FBE based model proposed in Ref. 3. To this aim, rather than applying a shear stress in random directions, we take benefit from the analysis of the DSC lattice, and its associated representation, the dichromatic pattern, in order to conceive judicious shear solicitation potentially able to activate alternative coupling modes.

In a first section, we explain the methodology to determine directions favoring alternative coupling modes. We then shear several symmetric tilt GB in $\mathrm{Cu}$ using Molecular Dynamics (MD) simulations in specific directions and evidence multiple coupling modes. We finally extend our study to an asymmetric [001]-tilt GB and a twist GB.

\section{Shear strain directions}

The SCGBM of perfect GBs occurs through the (homogeneous) nucleation and further motion of disconnections [29, 16, 13, 30, 14. Both processes are thermally activated. The SCGBM activation energy is dominated by the disconnections nucleation [13, 30. This nucleation activation energy barrier typically involves the disconnection core energies, the elastic disconnection-disconnection interaction energy and finally the Peach-Koehler energy due to the work of the shear stress. In the following, we consider a GB plane normal to the $x$ direction (see a sketch in Fig. 1), and apply a shear stress $\overline{\bar{\sigma}}\left(\sigma_{i i}=0\right.$ with $\left.i \in\{x, y, z\}\right)$. Glissile disconnections have thus, Burgers vectors perpendicular to $x$. Following [13, 30, 14, assuming a linear isotropic elastic crystal (Poisson ratio $\nu$, and shear modulus $\mu$ ), the energy interaction of a migrating GB displaying two (straight) disconnections with opposite Burgers vectors $\left(\vec{b}=b_{y} \vec{e}_{y}+b_{z} \vec{e}_{z}\right)$ separated by a

\footnotetext{
${ }^{1}$ The motion of a disconnection that would have a Burgers vector non parallel to the GB plane would imply climb and then long range atomic diffusion, which is not considered here.
} 
distance $\delta$ writes:

$$
E(\delta)=2 E_{\text {core }}\left(\delta_{0}\right)+\frac{\mu}{2 \pi(1-\nu)} \ln \frac{\delta}{\delta_{0}}-\left(\sigma_{x y} b_{y}+\sigma_{x z} b_{z}\right) \delta
$$

$\delta_{0}$ and $E_{\text {core }}\left(\delta_{0}\right)$ are the core radius and core energy of the disconnections. The activation energy barrier $E_{a c t}$ of the migration can be deduced from the maximum $E_{\max }$ of this energy $E(\delta)$ as a function of $\delta$.

$$
E_{\text {max }}=2 E_{\text {core }}\left(\delta_{0}\right)+\frac{\mu}{2 \pi(1-\nu)}\left[\ln \left(\frac{\mu}{2 \pi \delta_{0}(1-\nu)\left(\sigma_{x y} b_{y}+\sigma_{x z} b_{z}\right)}\right)-1\right]
$$

The maximum energy $E_{\max }$ is a decreasing function of the applied shear $\sigma_{x y}$ and $\sigma_{x z}$. Obviously, the application of a shear stress reduces the maximum energy and thus the activation energy barrier, and favors the occurrence of the migration [31, 8, 9. Eq. (1) and 22) can be written for every potential coupling modes (or disconnections). Single or multiple [3, 22] coupling modes can operate in order to accommodate the shear. In most studies [3, 32, the applied stress is a simple shear stress $\sigma_{x z}$ perpendicular to tilt axis in symmetric tilt GB, while in a real poly-crystal, the local shear stress can have any direction. This variety of potential stresses can presumably activate alternative disconnections: indeed, from Eq. (2), the nucleation of a given disconnection (and thus a given SCGBM coupling mode) will be favored if $E_{\max }$ is minimized, i.e. if the shear stress is applied in the direction of the Burgers vector of the disconnection. Note that for simplicity, we have considered a SCGBM induced by the motion of two infinite straight disconnections in Eq. (2), but considering the expansion of a disconnection loop would yield the same conclusion [19].

In the following, we identify the shear directions corresponding to Burgers vectors of mobile disconnections using the dichromatic pattern and shear a set of GBs in these directions.

\section{Molecular dynamics simulations}

We perform MD simulations in a FCC material using an embedded-atom potential for copper [33. We have investigated a series of [001] tilt GB with tilt angles ranging between $16.26^{\circ}$ and $73.74^{\circ}: \Sigma 25(710)\left(16.26^{\circ}\right)$, $\Sigma 37(610)\left(18.92^{\circ}\right), \Sigma 13(510)\left(22.62^{\circ}\right), \Sigma 17(410)\left(28.07^{\circ}\right), \Sigma 5(310)\left(36.87^{\circ}\right), \Sigma 5(210)\left(53.13^{\circ}\right), \Sigma 13(320)\left(67.38^{\circ}\right)$, $\Sigma 25(430)\left(73.74^{\circ}\right)$. In addition, we have also studied an asymmetric tilt GB $\Sigma 11(557)(771)[001]$ and a [001] pure twist GB $\Sigma 13(320)$. MD simulations have been performed at $0 \mathrm{~K}$ and in several cases at finite temperature.

Each of these GB has been studied using the same procedure. Figure 1 shows a sketch of the simulation cell in the case of a [001] tilt boundary (the cells for the asymmetric and twist GB are similar). The simulation cell contains two grains of a perfect FCC crystal disorientated relatively to each other around the [001] direction and separated by a perfect GB. Periodic boundary conditions (PBCs) are applied in the $\mathrm{y}$ - and z-directions. Typical simulation cell x-, y-, and z- sizes are, respectively, 10.8, 5.9, and 4.3 nm. The cell typically contains from about 30000 to 100000 atoms depending on the investigated GB. Equilibrium structures of the GB are obtained by minimization of the interface energy between the two grains and we have checked that they agree with GB structures reported in the literature. Two 1.7-nm-thick slabs at the top and bottom of the cell contain atoms with relative positions frozen to the perfect lattice ones. The slabs are translated relatively to each other parallel to GB plane by small increments in order to impose a shear stress on the GB. After each translation, a conjugate gradient algorithm minimizes the potential energy during which slabs can move freely in the x-directions. Elementary mechanisms of GB migrations are determined following the procedure reported in Ref. [13: from the configurations before and after the GB migration at a given shear strain, the disconnections involved during the GB migration are evidenced from Nudge Elastic Band (NEB) calculations [34. In addition, on few GBs, some simulations of GB migration at finite temperature were performed using a Nose Hoover thermostat to check the robustness of our findings. All simulations have been performed using the LAMMPS simulations sofware [35, 36. Below, in order to avoid a fastidious catalog of the observed GB migration for all investigated GB and all shear directions, we have chosen to detail our results for two symmetric tilt GBs $\Sigma 17(410)[001]$ and $\Sigma 13(320)[001])$, an asymmetric 


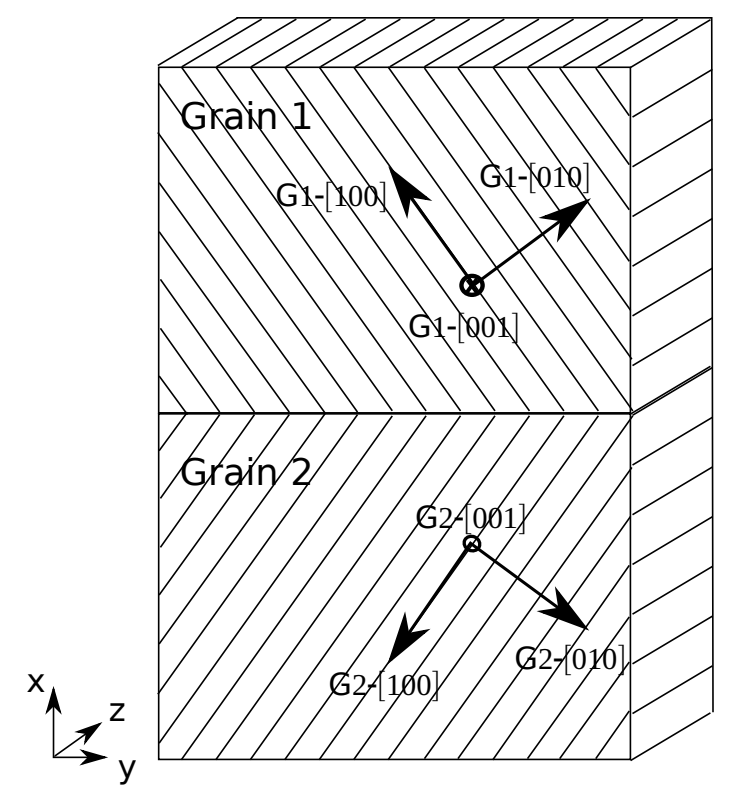

Figure 1: (color online) Sketch of the simulated system. A GB perpendicular to the x direction separates two grains $G_{1}$ and $G_{2}$.

tilt GB $\Sigma 11(557)(771)[001]$ and a pure twist GB $\Sigma 13[001]$. Results for other tilt GBs will be synthesized in a figure.

\section{Disconnection notation}

The notation commonly used to designate a disconnection is the so-called $p / q$ notation introduced in Ref. 37]: $p$ and $q$ are related to the step height in each grain (perfect lattice). Unfortunately, this notation, though very simple is ambiguous because several different disconnections have the same p/q notation. In the present study, we need to unambiguously designate a disconnection. We thus propose a notation based on the crystal lattice coordinate systems (Fig. 1). In the following we propose to identify a disconnection by the triplets $\left(n_{1} m_{1} p_{1}\right) /\left(n_{2} m_{2} p_{2}\right)$ : the Burgers vector $\vec{b}_{\left(n_{1} m_{1} p_{1}\right) /\left(n_{2} m_{2} p_{2}\right)}$ and step height $h_{\left(n_{1} m_{1} p_{1}\right) /\left(n_{2} m_{2} p_{2}\right)}$ of this disconnection are respectively:

$$
\begin{aligned}
\vec{b}_{\left(n_{1} m_{1} p_{1}\right) /\left(n_{2} m_{2} p_{2}\right)} & =\vec{t}_{2}\left(n_{2} m_{2} p_{2}\right)-\vec{t}_{1}\left(n_{1} m_{1} p_{1}\right) \\
h_{\left(n_{1} m_{1} p_{1}\right) /\left(n_{2} m_{2} p_{2}\right)} & =\frac{1}{2}\left(\vec{t}_{1}\left(n_{1} m_{1} p_{1}\right)+\vec{t}_{2}\left(n_{2} m_{2} p_{2}\right)\right) \cdot \vec{u}_{x}
\end{aligned}
$$

where $\vec{t}_{i}(n m p)$ refers to the vectors expressed in grains $i$ :

$$
\vec{t}_{i}(n m p)=\frac{1}{2}\left[n_{i}, m_{i}, p_{i}\right]
$$

and $\vec{u}_{x}$ is a unitary vector normal to the GB. The factor $\frac{1}{2}$ in Eq. (4) could have been eluded, but it avoids numerous fractional numbers when designating disconnections in the following. We adopt this notation $\left(n_{1} m_{1} p_{1}\right) /\left(n_{2} m_{2} p_{2}\right)$ to designate a disconnection in this manuscript. But in order to avoid a confusing notation for readers familiar with the common $p / q$ notation, we also mention this latter one in bracket when designating a disconnection. Note that the introduced notation (as the $p / q$ one) does not provide any information on the core structure of the disconnection. 


\section{Results}

\section{1. $\Sigma 17(410)-[001]$}

We first explicit below our finding for the $\Sigma 17(410)$ - [001] symmetric tilt GB. Fig. 1 1 shows a sketch of the proposed system with $\mathrm{x}, \mathrm{y}$ and $\mathrm{z}$ axis respectively along [410], [140] and [001] crystal (grain 1) directions. Fig. 2 shows the dichromatic pattern of the $\Sigma 17(410)$ - [001] symmetric tilt GB and the grain orientation.

\subsubsection{Disconnection and shear direction}

Figure 2 shows numerous potential disconnections labeled using the notation introduced above. All these disconnections have Burgers vectors parallel to the GB plane and are thus glissile. Each of these disconnections can be related to a disconnection with opposite Burgers vectors and step height (associated to the same coupling mode): for clarity, we have chosen to mention only disconnections with positive Burgers vector y-components.

Let's first focus on the $(101) /(\overline{1} 01)[4 / 4]$ and $(\overline{1} \overline{1} 0) /(110)[-5 /-5]$ disconnections. The glide of such disconnections respectively induces a GB migration under the so-called $\langle 100\rangle$ and $\langle 110\rangle$ modes 3 , 8. The Burgers vector norm (step height) of the disconnections (101)/(101) $[4 / 4]$ and $(\overline{1} 10) /(110)[-5 /-5]$ are respectively $0.088 \mathrm{~nm}(0.175 \mathrm{~nm})$ and $0.26 \mathrm{~nm}(0.22 \mathrm{~nm})$. Since the Burgers vectors $\vec{b}_{(101) /(101)[4 / 4]}$ and $\vec{b}_{(\overline{1} \overline{1} 0) /(110)[-5 /-5]}$ are both parallel to the y-direction, the most efficient shear stress to activate these modes is given by $\sigma_{x y} \neq 0\left(\sigma_{x z}=\sigma_{y z}=0\right)$, which is indeed verified [3]. The activation barriers of these two modes decrease as a function of an applied pure shear stress: the occurrence of a given mode depends on the relative values of the activation barriers of these modes [8.

Let's now consider the glissile disconnection $\vec{b}_{(\overline{2} 11) /(130)[-7 /-7]}$. Its Burgers vector norm, $0.28 \mathrm{~nm}$, and step height, $0.31 \mathrm{~nm}$, are comparable to those of the $(\overline{1} 10) /(110)[-5 /-5]$ disconnection, ie. $0.26 \mathrm{~nm}$ and 0.22 $\mathrm{nm}$, respectively. Hence, the activation energies for the $(\overline{2} 11) /(130)[-7 /-7]$ and $(\overline{1} \overline{1} 0) /(110)[-5 /-5]$ modes are expected to be of the same order of magnitude [14. However, to our knowledge, the $(\overline{2} 11) /(130)[-7 /-7]$ mode has never been evidenced experimentally nor numerically.

The shear stress that is the most efficient to activate the nucleation of the disconnection $(\overline{2} 11) /(130)[-7 /-$ 7 ] at $0 \mathrm{~K}$, has the direction of its Burgers vector.

\subsubsection{The example of the $(\overline{2} 11) /(130)[-7 /-7]$ shear direction}

The $\Sigma 17(410)-[001]$ symmetric tilt GB is sheared at $0 \mathrm{~K}$ in a direction parallel to $\vec{b}_{(\overline{2} 11) /(130)[-7 /-7]}$. Note that the Burgers vector $\vec{b}_{(\overline{2} 11) /(130)[-7 /-7]}$ has a screw-component, i.e. along the [001] tilt axis (z-axis). During the shear, we observe a stick slip displacement of the GB evidencing a succession of elastic and plastic regimes. Fig. 3h shows the shear stresses $\sigma_{x y}$ and $\sigma_{x z}$ as a function of the y-slab displacement. Each stress drop corresponds to a plastic event, here a GB migration. This behavior is consistent with previous numerical results [3, 13]. These curves suggest two kind of GB migration: type i) occurs at $0.17 \mathrm{~nm}$ y-slab displacement, and type ii) occurs at $0.31 \mathrm{~nm}, 0.41 \mathrm{~nm}$ and $0.52 \mathrm{~nm} .$.

For a reason that will appear below, we first focus on type ii) GB migration. Fig. 3 3 p shows the atomic configurations along the minimum energy path (MEP) evidencing the disconnections that homogeneously nucleate during this type ii) GB migration. This disconnection actually corresponds to the $(\overline{2} 11) /(130)[-7 /-7]$ as can be revealed by a Burgers circuit analysis. The nucleation of this disconnection is also corroborated by the stress-strain curve: the successive yield stresses $\sigma_{x y}$ and $\sigma_{x z}$ for all type ii) GB migrations are equal (except small variations due to finite sizes of the simulations cells): this coupling mode exactly dissipates the elastic energy accumulated while displacing the slabs in the direction $\vec{b}_{(\overline{2} 11) /(130)[-7 /-7]}$. Analyzing the type i) GB migration using the same method, we evidence the nucleation of a $\vec{b}_{(\overline{1} 10) /(031)[-3 /-3]}$ disconnection that was not expected. The Burgers vector norms (and step height) of the $(\overline{110}) /(031)[-3 /-3]$ and $(\overline{2} 11) /(130)[-7 /-7]$ being respectively $0.35 \mathrm{~nm}(0.22 \mathrm{~nm})$ and $0.28 \mathrm{~nm}(0.31 \mathrm{~nm})$, the core energy of the $(\overline{1} 10) /(031)[-3 /-3]$ and $(\overline{2} 11) /(130)[-7 /-7]$ disconnections are expected to be of the same order of magnitude [14. But more important, the activation energy of the disconnections nucleation depends on both shear stress components $\sigma_{x y}$ and $\sigma_{x z}$ : the nucleation of disconnections $(110) /(031)[-3 /-3]$ occurs for yield stresses $\sigma_{x y}=1.72 G P a$ and $\sigma_{x z}=1.58 G P a$ and allows to accommodate $90 \%$ of the shear stress along xz 


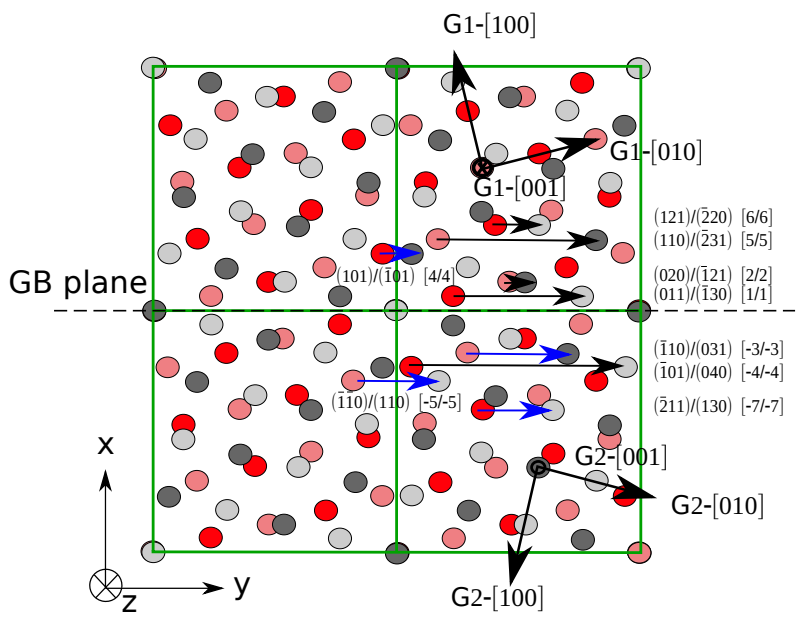

Figure 2: (color online) Projection of the dichromatic pattern of the $\Sigma 17(410)$ - [001] GB along the [001] z-direction reporting the shearing direction (black and blue arrows) and the observed disconnections (blue arrows). The GB plane is reported by a dashed black line. Black (Grey) and Red (Pink) atoms belong to different grains. Black(Red) and Grey(Pink) atoms have not the same z-coordinate. Green solid lines correspond the coincident lattice site cell.
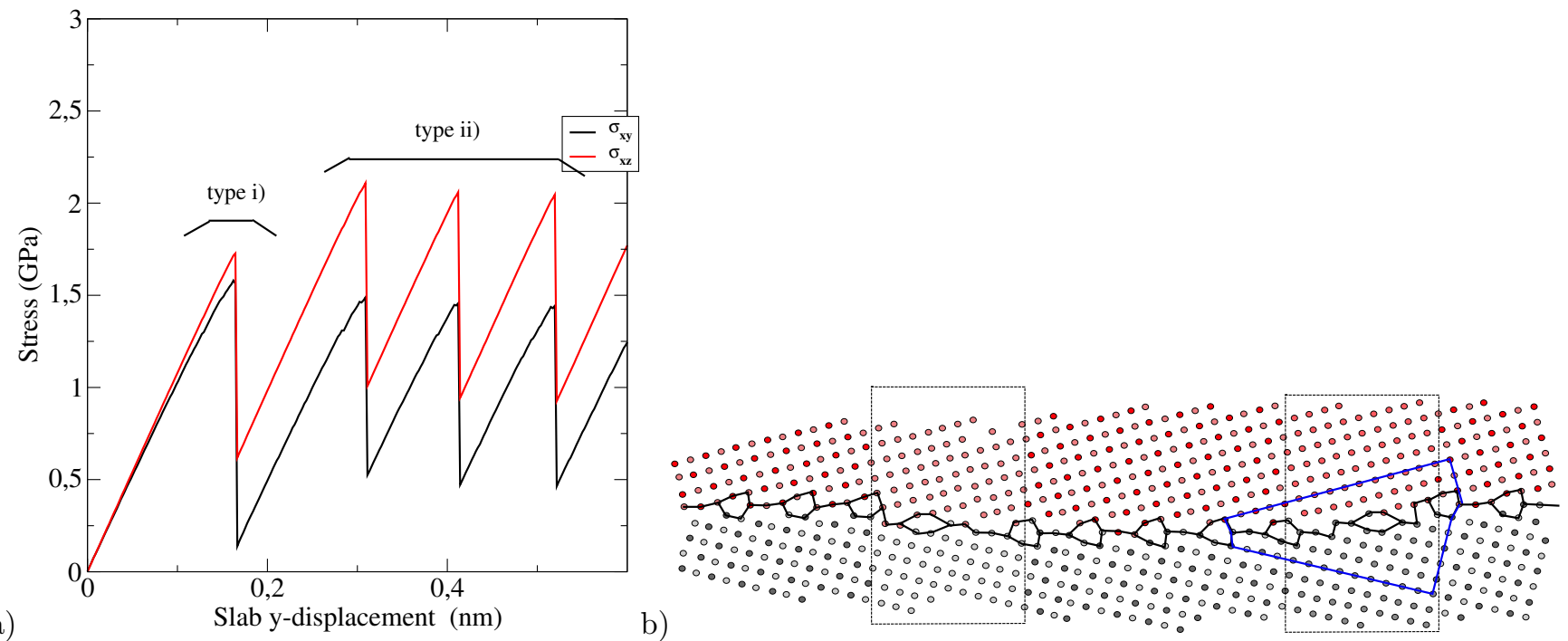

Figure 3: (color online) a) Shear stress-strain curve $\Sigma 17(410)$ - [001] as a function of the y-slab position when shearing along the $\vec{b}_{(\overline{2} 11) /(130)[-7 /-7]}$. b) Disconnections $(\overline{2} 11) /(130)[-7 /-7]$ in the $\Sigma 17(410)-[001]$ symmetric tilt GB corresponding to the atomic configuration along the MEP at reaction coordinate 1 The reaction coordinate is defined as the cumulative distance (normalized by the total cumulative distance) between adjacent replicas in the configuration space of dimension $3 \mathrm{~N}$ with $\mathrm{N}$ the number of atoms 0.73 (the slab displacement is $0.17 \mathrm{~nm}$ ). The black solid lines, a guide to the eyes join atomic rows to reveal the structural units. A Burgers circuit around the right disconnection is reported as a blue solid line. The atom color code is identical to the one of Fig. $2 \mathrm{p}$ excepted in the region delimited by a dashed rectangle. In this region, a uniform atom color code has been used: indeed, the screw component of the Burger vectors of disconnections $(\overline{2} 11) /(130)$ imposes a relative displacement of grain 1 compared to grain 2 in the direction [001].

but only $63 \%$ of the one along xy. While both shear stress components $\sigma_{x y}$ and $\sigma_{x z}$ were almost equal before the operation of the disconnection $(\overline{1} 10) /(031)[-3 /-3]$, the operation of this latter one induces a smaller $\sigma_{x z}$ drop than the $\sigma_{y z}$ : this regime is transitional. The nucleation of $(\overline{2} 11) /(130)[-7 /-7]$ disconnections then operate with yield stresses $\sigma_{x y}=2.10 \mathrm{GPa}$ and $\sigma_{x z}=1.49 \mathrm{GPa}$ and lead to a stationary stick-slip regime. 


\subsubsection{Other directions}

We also tried to activate other disconnections by shearing in the direction of the $(011) /(\overline{1} 30)[1 / 1]$, $(020) /(\overline{1} 21)[2 / 2],(\overline{1} 10) /(031)[-3 /-3],(\overline{1} 01) /(040)[-4 /-4],(110) /(\overline{2} 31)[5 / 5)],(121) /(\overline{2} 20)[6 / 6]$ disconnections Burgers vectors at $0 \mathrm{~K}$ : all these shear directions are reported in the dichromatic pattern in Fig. 2.

For numerous cases, we have observed a shear coupled GB migration under the expected or an alternative coupling mode or a periodic succession of different coupling modes. The GB structure is conserved during this GB migration and hence, the nucleated disconnections can be characterized. But we have also observed shear coupled GB migration mechanisms during which the GB structure is not conserved and beyond, the GB sometimes does not remain planar. In such cases, the characterization of the disconnections and especially the elaboration of a relevant Burgers circuit is tricky: to our knowledge, a recent method of disconnections analysis available in this case has been reported in the literature [38. GB defect between two different GB structure can be characterized by a Burgers vectors $\vec{b}_{D S C}+\vec{b}_{\text {defect }}$ where $\vec{b}_{D S C}$ and $\vec{b}_{\text {defect }}$ respectively do and do not belong to the DSC lattice. Because GB migration conserving the GB structure are numerous in our study, we have focused on them and did not try to address cases with GB structures changes. In the following, we report only results concerning cases where the GB structure is conserved and when disconnections can be analyzed.

Tab. 1 summarizes all the disconnections observed in the NEB calculations while investigating the $\Sigma 17(410)-[001]$ GB.

By varying the shear directions, the disconnections $(\overline{1} 10) /(031)[-3 /-3],(101) /(\overline{1} 0 \overline{1})[4 / 4],(\overline{1} 10) /(110)[-5 /-$ $5],(\overline{2} 11) /(130)[-7 /-7]$ have been activated. These disconnections are reported with blue arrows in Fig. 2 . Note that though their $p / q$ notations are identical, the $(101) /(\overline{1} 0 \overline{1})[4 / 4]$ disconnection differs from the $(101) /(\overline{1} 01)[4 / 4]$ one (mentioned previously as operating under the $\langle 100\rangle$ coupling mode). Indeed, the $(101) /(\overline{1} 0 \overline{1})[4 / 4]$ Burgers vector has a screw component along the [001] direction, while the $(101) /(101)[4 / 4]$ has not. Note that since Fig. 2 shows the projection of the dichromatic pattern on the [001] direction, both disconnections $(101) /(\overline{1} 0 \overline{1})[4 / 4]$ and $(101) /(\overline{1} 01)[4 / 4]$ superimpose on Fig. 2 .

When shearing along the $(\overline{1} 10) /(031)[-3 /-3]$ and $(\overline{2} 11) /(130)[-7 /-7]$ directions, the coupling modes and disconnections $(\overline{1} 10) /(031)[-3 /-3]$ and $(\overline{2} 11) /(130)[-7 /-7]$ are activated and accordingly allow to relax the applied shears.

Surprisingly, when shearing at 0K along the $(\overline{1} 10) /(231)[5 / 5],(011) /(\overline{1} 30)[1 / 1]$ or $(\overline{1} 01) /(040)[-4 /-4]$ directions, none of the observed disconnections $(\overline{1} \overline{1} 0) /(110)[-5 /-5]$ and $(\overline{1} 10) /(031)[-3 /-3]$ have their Burgers vectors parallel to the applied shear stress. In these cases, the shear stress is relaxed by a periodic succession of several coupling modes: an illustrative example of such a case will be detailed below in the case of the $\Sigma 13(320)$ GB. Finally, when shearing along the $(121) /(\overline{2} 20)[6 / 6]$ directions, several elementary GB migrations reported in the Tab. 1 are first observed before the GB changes its structure.

\subsubsection{Finite temperature simulations}

Several MD simulations at $100 \mathrm{~K}$ and $200 \mathrm{~K}$ have been performed. They have evidenced the nucleation of the $(\overline{1} \overline{1} 0) /(031)[-3 /-3]$ and $(\overline{2} 11) /(130)[-7 /-7]$ disconnections in the $\Sigma 17(410)-[001]$ GB. However, apart from few simulations that have allowed an observation and characterization of the nucleated disconnections, the GB structure is most often not conserved during the SCGBM yielding to the impossibility to directly analyze the elementary mechanism in terms of disconnections. However, we emphasize that we have always observed a shear coupled GB migration, though we did not try to characterize this coupling in term of elementary mechanisms.

\section{2. $\Sigma 13(320)-[001]$}

Fig. 4 a shows the dichromatic pattern of the $\Sigma 13(320)-[001]$ GB and several potential glissile disconnections (black and blue arrows): we have investigated the shear of this GB in all the Burgers vectors directions of these disconnections at 0K. The nucleation of the $(020) /(\overline{2} 11)[4 / 4],(\overline{1} \overline{1} 0) /(110)[-5 /-5]$ and $(101) /(\overline{1} 0 \overline{1})[3 / 3]$ disconnections reported by blue arrows in Fig. 4 a have been observed. Note again that the $(101) /(\overline{1} 0 \overline{1})[3 / 3]$ coupling mode differs from the $(100) /(\overline{1} 00)[3 / 3]$ (operating during the $\langle 100\rangle$ coupling mode) since it has a screw component (along the [001] axis). 


\begin{tabular}{|c|cc|}
\hline Shear direction & Characterized Disconnections & $([\mathrm{p} / \mathrm{q}]$ notation $)$ \\
\hline \hline$(011) /(\overline{1} 30)[1 / 1]$ & $(\overline{1} \overline{1} 0) /(110)$ & {$[-5 /-5]$} \\
& $(\overline{1} 10) /(031)$ & {$[-3 /-3]$} \\
\hline$(\overline{1} 10) /(031)[-3 /-3]$ & $(\overline{1} 10) /(110)$ & {$[-5 /-5]$} \\
& $(\overline{2} 11) /(130)$ & {$[-7 /-7]$} \\
& $(\overline{1} 10) /(031)$ & {$[-3 /-3]$} \\
\hline$(\overline{1} 01) /(040)[-4 /-4]$ & $(\overline{1} 10) /(110)$ & {$[-5 /-5]$} \\
& $(\overline{1} 10) /(031)$ & {$[-3 /-3]$} \\
\hline$(110) /(\overline{2} 31)[5 / 5]$ & $(\overline{1} 10) /(110)$ & {$[-5 /-5]$} \\
& $(\overline{1} 10) /(031)$ & {$[-7 /-7]$} \\
\hline$(121) /(\overline{2} 20)[6 / 6]$ & $(\overline{2} 11) /(130)$ & {$[4 / 4]$} \\
& $(101) /(\overline{1} 0 \overline{1})$ & {$[-7 /-7]$} \\
& $(\overline{2} 11) /(130)$ & {$[-5 /-5]$} \\
\hline$(\overline{2} 11) /(130)[-7 /-7]$ & $(\overline{1} 10) /(031)$ & \\
\hline
\end{tabular}

Table 1: Summery of the $\Sigma 17(410)$ - [001] GB behavior depending on the shear direction. All observed and characterized disconnections are reported.

a)

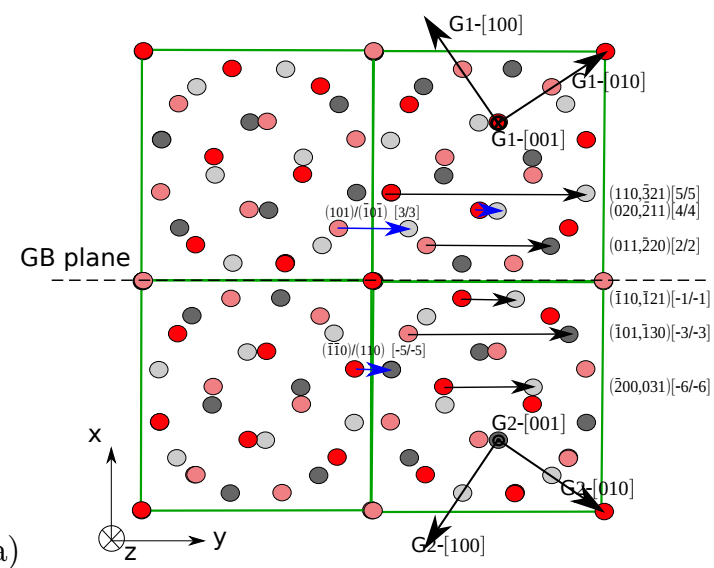

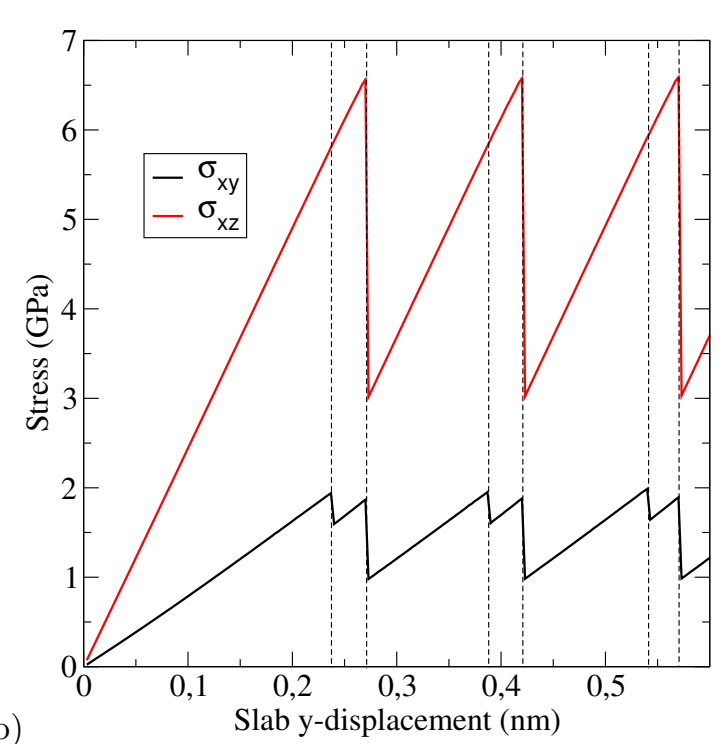

b)

Figure 4: (color online) a) Projection of the dichromatic pattern of the $\Sigma 13(320)$ - [001] along the [001] z-direction reporting the shearing direction (black and blue arrows) and the observed disconnections (blue arrows). The GB plane is in the (yz) plane. Same atoms color code as in Fig. 2 b) Shear stress-strain curve $\Sigma 13(320)-[001]$ as a function of the y-slab position when shearing along the $\vec{b}_{(\overline{1} 10) /(\overline{1} 21)[-1 /-1]}$. 
Below we detail an interesting behavior previously mentioned: the successive operation of different disconnections.

Fig. 4b shows the stress-strain curve measured when shearing the $\Sigma 13(320)-[001] \mathrm{GB}$ at $0 \mathrm{~K}$ along the $(110) /(121)[-1 /-1]$ direction. A periodic succession of two types of plastic events operates. A first one (in Fig. $4 \mathrm{~b}$ at $0.24 \mathrm{~nm}, 0.39 \mathrm{~nm}, 0.54 \mathrm{~nm} .$. ) induces a drop in the $\sigma_{x y}$ stress but no $\sigma_{x z}$ drop : the examination of this event using the NEB method shows the operation of $(\overline{1} \overline{1} 0) /(110)[-5 /-5]$ disconnections (corresponding to the operation of $\langle 110\rangle$ coupling mode). Accordingly, the absence of component along the z-direction of the Burgers vector of the disconnection $(\overline{1} 10) /(110)[-5 /-5]$ agrees with the absence of $\sigma_{x z}$ stress drop. The corresponding coupling factor is negative. The second plastic event (at $0.27 \mathrm{~nm}, 0.42 \mathrm{~nm}, 0.57 \mathrm{~nm} .$.$) induces$ stress drops for both $\sigma_{x y}$ and $\sigma_{x z}$ : the examination of this event using the NEB method reports the operation of the $(101) /(\overline{1} 0 \overline{1})[3 / 3]$ disconnection. The Burgers vector of such disconnections have both a component on the $\mathrm{y}$ - and the $\mathrm{z}$-directions allowing the relaxation of the stress in both directions. The corresponding coupling factor is positive. The combination of these two plastic events $(\overline{1} \overline{1} 0) /(110)[-5 /-5]$ and $(101) /(\overline{1} 0 \overline{1})[3 / 3]$ fully accommodates the shear stresses when shearing along $(\overline{1} 10) /(\overline{1} 21)[-1 /-1]$. Indeed, we note:

$$
\begin{aligned}
\vec{b}_{(\overline{1} \overline{1} 0) /(110)[-5 /-5]}+\vec{b}_{(101) /(\overline{1} 0 \overline{1})[3 / 3]} & =2 \vec{b}_{(\overline{1} 10) /(\overline{1} 21)[-1 /-1]} \\
h_{(\overline{1} 10) /(110)[-5 /-5]}+h_{(101) /(10 \overline{1})[3 / 3]} & =2 h_{(\overline{1} 10) /(\overline{1} 21)[-1 /-1]}
\end{aligned}
$$

This relation can be derived from the sum of the coefficients

$$
(\overline{1} \overline{1} 0) /(110)[-5 /-5]+(101) /(\overline{1} 0 \overline{1})[3 / 3]=(0 \overline{1} 1) /(01 \overline{1})[-2 /-2]
$$

moreover, invoking $(\overline{2} 3 \overline{1}) /(\overline{2} 33)[-13 / 0]$ a vector of the coincident site lattice, we get:

$$
\begin{aligned}
(0 \overline{1} 1) /(01 \overline{1})[-2 /-2]+(\overline{2} 3 \overline{1}) /(\overline{2} 33)[-13 / 0] & =(\overline{2} 20) /(\overline{2} 42)[-2 /-2] \\
& =2(\overline{1} 10) /(\overline{1} 21)[-1 /-1]
\end{aligned}
$$

Hence, even at $0 \mathrm{~K}$, the SCGBM can successively involve several different elementary events, each of them associated to a disconnection and thus a coupling factor. Surprisingly, the imposed shear is not relaxed by the single $(\overline{1} 10) /(\overline{1} 21)[-1 /-1]$ mode (and its corresponding disconnection). This result has already been established at finite temperature: SCGBM is a thermally activated process so that different coupling modes can compete with each other [3, 8, 22. Here, we extend it to the low temperature case. While in the literature, most authors, including us, used to believe that at low temperature, the disconnection mode with the lowest energy barrier was the only activated one [13, 8, 22, our present results precise this assertion: several disconnections can compete with each other even at low temperature since the activation energy is a multivariate function of the shear stresses $\sigma_{x y}$ and $\sigma_{x z}$.

Since the coupling factors (along the y-direction) of the $(\overline{1} \overline{1} 0) /(110)[-5 /-5]$ and $(101) /(\overline{1} 0 \overline{1})[3 / 3]$ disconnections are opposite and their values are respectively $\beta_{[-5 /-5]}=\frac{b_{y}[-5 /-5]}{h[-5 /-5]}=-0.399$ and $\beta_{[3 / 3]}=\frac{b_{y}[3 / 3]}{h[3 / 3]}=$ 1.33, the effective coupling factor is $\beta_{e}=\frac{b_{y}[-5 /-5]+b_{y}[3 / 3]}{h[-5 /-5]+h[3 / 3]}=3.002$ If we relate this value to experimental measurements, the successive operation of multiple coupling modes seems not to be a relevant argument to explain the low coupling factors reported by experiments on poly-crystalline metals.

\subsection{Symmetric [001]-tilt GB: a synthesis}

Fig. 5 presents a synthesis of our results on all investigated [001] tilt GBs. Fig. 5 shows the misorientation angle of the GB as a function of the coupling factors $\beta_{y}=\frac{b_{y}}{h}$ and $\beta_{z}=\frac{b_{z}}{h}$ of the disconnections that we have evidenced. Both solid lines mention the $\langle 100\rangle$ and $\langle 110\rangle$ coupling modes derived from the FBE [3]. Fig. 5] clearly evidences that the GB disposes of numerous coupling modes to accommodate the shear stress. The $\langle 100\rangle$ and $\langle 110\rangle$ coupling modes only represent a small fraction of available coupling modes. This conclusion is general and applies for all investigated GBs.

${ }^{2}$ Note that the effective factor is not the average nor a linear combination of coupling factors 


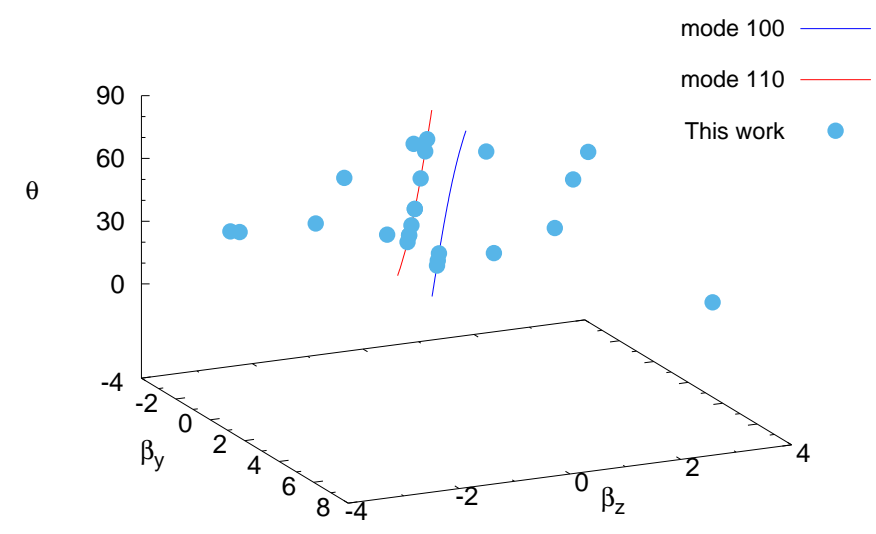

Figure 5: Misorientation angle of the investigated tilt-[001] GBs as a function of the coupling factors $\beta_{y}=\frac{b_{y}}{h}$ and $\beta_{z}=\frac{b_{z}}{h}$ of the evidenced disconnections. Blue and Red solid lines draw the $\langle 100\rangle$ and $\langle 110\rangle$ coupling modes as described in [3].

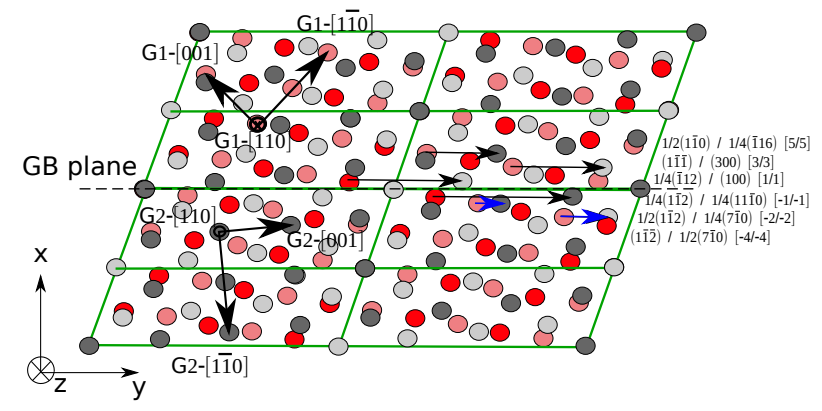

Figure 6: Projection of the dichromatic pattern of the $\Sigma 11(557)(771)$ - [001] GB along the [001] z-direction reporting the shear direction (black and blue arrows) and the observed disconnections (blue arrows). The GB plane is in the (yz) plane. Same atoms color code as in Fig. 2 For clarity, two CSL cell have been shown.

\subsection{Asymmetric $G B: \Sigma 11(557)(771)[110]$}

In order to show the generality of our procedure to generate alternative coupling modes, we have considered an asymmetric GB $\Sigma 11(557)(771)-[110]$ which dichromatic pattern is showed in Fig. 6. The response of $\Sigma 11(557)(771)-[110]$ GB to a pure shear stress $\sigma_{x y}>0$ has been previously studied by Zhang et al. [39: Zhang et al. showed that the deformation mechanism consisted in the emission of the dissociated partial dislocations and the nucleation and propagation of the partial dislocations from the GB plane. They did not report that the GB migrates and couples with the shear. We found the same results. However, applying a negative component $\sigma_{x y}<0$ of the shear stress, we observed a GB migration coupled to the shear. We have thus investigated numerous shear stress directions (all of them involve a negative value of the xy shear stress component $\sigma_{x y}<0$ ) corresponding to the glissile disconnections reported in Fig. 6. Two coupling modes corresponding to the disconnections with Burgers vectors indicated by blue arrows in the dichromatic patterns were observed. In some cases, a periodic succession of these two coupling modes similarly to our observations for the $\Sigma 13(320)$ - [001] tilt GB were evidenced.

\subsection{Twist GB: $\Sigma 13(001)$}

Finally, we would like to address a question hardly mentioned in the literature: does a twist GB shear couple? Fig. 7 $\mathrm{a}$ ) and b) show the dichromatic patterns of the $\Sigma 13(001)$ twist GB projected along [001] (x direction) and [320] (z direction), respectively. Lattice planes above and below the GB plane are identical 


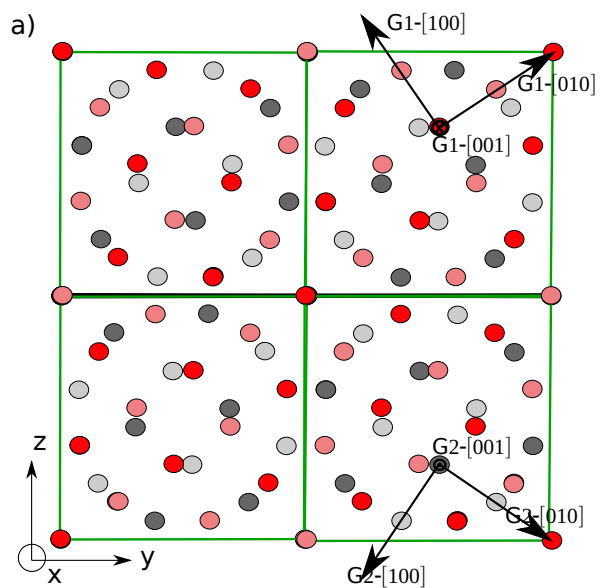

b)

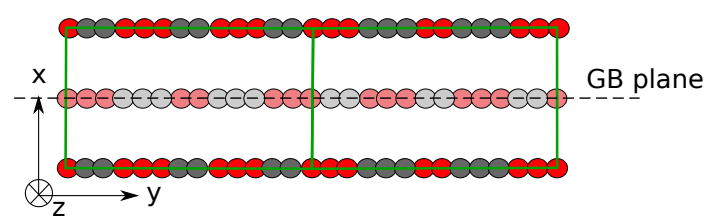

Figure 7: a) and b) Projections of the dichromatic pattern of the twist $\Sigma 13(320)-(001)$ GB along the [001] direction (x direction) and the [320] y-direction. Same atoms color code as in Fig. 2 .

in the dichromatic pattern, because (001) is a mirror plane of the DSC. Hence, applying a shear stress (parallel to the GB plane) in any direction, is not expected to lead to GB migration since its migration in the opposite direction is totally equivalent: for any potential glissile disconnections of Burgers vector $\vec{b}$ and step height $h$, there exists a disconnection of Burgers vectors $\vec{b}$ and step height $-h$. However, GB sliding an operate: there exists disconnections with Burgers vectors $\vec{b} \neq \overrightarrow{0}$ and no step heigh $h=03$ Applying a shear stress on a twist GB, we thus expect that the average position 4 of the GB will not evolve with time, and that the shear elastic energy will be relaxed by a sliding of the two grains. We have performed MD simulations at $0 \mathrm{~K}$ to shear the twist $\Sigma 13(320)-(001)$ GB in directions parallel to various disconnection Burgers vectors parallel to the GB plane. We have actually observed some sliding without migration. Seldom, we have observed some fluctuations of the GB position around its average position. This behavior is reminiscent of the random nucleation of disconnections with identical Burgers vector but with opposite heights $h= \pm 0.18 \mathrm{~nm}$ as expected.

\section{Discussion and conclusion}

Investigating numerous GB types (symmetric, asymmetric, twist) and numerous shear directions, this work clearly highlights that SCGBM generally operates through the motion of disconnections. The operation of a given disconnection can be tentatively predicted and favored by imposing a shear stress in the Burger vector direction. This approach combining geometry, local stress field distribution and disconnections nucleation energetic considerations is thus expected to provide a description of the coupling modes at the grain scale.

At the beginning of this manuscript, we mentioned that the coupling factor for SCGBM has been previously explained by: 1) modeling the GB by an intrinsic dislocation network using the FBE and 2) predicting the coupling factor from the glide of these dislocations [3. As mentioned previously, for a symmetric tilt

\footnotetext{
${ }^{3}$ Note that some authors reserve the term disconnection for GB defects with $h \neq 0$ and $\vec{b} \neq \overrightarrow{0}$.

${ }^{4}$ We refer here to a statistical average.
} 
GB in a FCC crystal, this model predicts four potential coupling modes [3] in disagreement with our present results (evidencing the operation of numerous disconnections/coupling modes). However, as previously noticed [4, and as a consequence of the FBE multiplicity, several intrinsic dislocation networks can describe a given GB. Any of the coupling modes we have evidenced above can be explained by one of these descriptions 4. In that sense, both dislocation and disconnection models are completely equivalent from a geometrical point of view: both deals with transport of a shear either using an intrinsic dislocation or a disconnection formalism. Note that pure geometric models [40, 41] equivalent to these two models have also been elaborated.

We think that the disconnections model is more physical to describe the SCGBM . Indeed, the SCGBM effectively operates through the nucleation and motion of disconnections. Moreover, the disconnections model relies on the dichromatic pattern, a characteristic of the GB that includes all the potential disconnections and equally describes them. Hence, we agree with the conclusion of Ref. 22 that the SCGBM is not an intrinsic property of the GB. In that sense, GB based plasticity is not different from dislocation based crystal plasticity, except the variety of disconnections has to be taken into account.

Another important conclusion of the present work is that the coupling factor measured at the meso-scale is not an intrinsic property of the underlying elementary mechanisms (disconnection nucleation and motion), even at very low temperature. Hence, an investigation of the GB migration at the atomic scale in order to characterize disconnections is necessary to raise a reliable conclusion on the elementary mechanisms operating during a GB migration. The analysis of the elementary processes of the GB migration is unfortunately difficult at both high and low temperatures both experimentally and numerically. Experimentally, only in situ HRTEM have the required atomic scale resolutions 21. But these experiments are technically highly challenging and require ultra fine samples where diffusion effects at free surfaces need to be taken into account. Numerically, two main issues restrains the analysis of the elementary mechanisms: first, GB based plasticity can involve several elementary mechanisms that may operate simultaneously and potentially cooperatively [42. Second, the atomic structures of GBs can evolve during their migration and to our knowledge, there is no method to analyze disconnections in these cases. The understanding and knowledge of GB based plasticity would highly benefit from significant advances on these two issues.

In this manuscript, we have focused on shear directions that could be accommodated by a disconnection present in the dichromatic pattern. But we have also tentatively investigated several general shear direction corresponding to none of the disconnection displayed in the dichromatic pattern. We have most often observed the operation of the disconnections that accommodate most but not totally the shear and then, a destabilization of the GB yielding a rough GB caused mainly by large fluctuation of the GB plane. The rough GB then migrates in order to accommodate the stress. We think that in rough GB, the presence of multiple step creates a lot of potential nucleation sources of mobiles disconnections allowing presumably the GB to relax the elastic energy through the operation of different types of disconnections. Unfortunately, though this interpretation seems reasonable, identification of GB steps in rough GB is far from obvious so that a definitive conclusion can not be raised from our present simulations. It seems however rather clear that a perfectly flat GB has less channels to relax the elastic energy than a rough GB. The understanding of the migration of rough GBs is clearly an important research perspective for the GB based plasticity.

Finally, we have limited the present study to homogeneous disconnections nucleation and have observed the operation of numerous disconnections (or coupling mode). But numerous other potential disconnections (the one represented by black arrows) present in the dichromatic patterns did not activate. These latter ones may be activated by heterogeneous disconnection nucleation: vacancies 43, 44, triple junctions [45, sessile disconnections [46, 47] or other GB imperfections can be a source of glissile disconnections and could potentially produce disconnections that have not been observed.

This work was performed using HPC resources from CALMIP (Grant No. 2012-12172) and was funded by ANR-17-CE08-0007.

[1] D. Gianola, S. V. Petegem, M. Legros, S. Brandstetter, H. V. Swygenhoven, K. Hemker, Stress-assisted discontinuous grain growth and its effect on the deformation behavior of nanocrystalline aluminum thin films, Acta Materialia 54 (8) (2006) 2253 - 2263. doi:10.1016/j.actamat.2006.01.023

[2] A. Sutton, R. W. Balluffi, Interfaces in crystalline materials, Oxford:Clarendon Press ; New York : Oxford University Press, 1995 
[3] J. W. Cahn, Y. Mishin, A. Suzuki, Coupling grain boundary motion to shear deformation, Acta Mat. 54 (2006) $4953-4975$. doi: $10.1016 / j$.actamat .2006 .08 .004

[4] K. D. Molodov, D. A. Molodov, Grain boundary mediated plasticity: On the evaluation of grain boundary migration shear coupling, Acta Materialia 153 (2018) 336 - 353. doi:10.1016/j.actamat.2018.04.057

[5] W. T. Read, W. Shockley, Dislocation models of crystal grain boundaries, Phys. Rev. 78 (1950) 275-289. doi:10.1103/ PhysRev.78.275

[6] J. W. Cahn, Y. Mishin, A. Suzuki, Duality of dislocation content of grain boundaries, Phil. Mag, 86 (2006) 3965. doi: $10.1080 / 14786430500536909$

[7] T. Frolov, Effect of interfacial structural phase transitions on the coupled motion of grain boundaries: A molecular dynamics study, Applied Physics Letters 104 (21) (2014) 211905. doi:10.1063/1.4880715.

[8] N. Combe, F. Mompiou, M. Legros, Disconnections kinks and competing modes in shear-coupled grain boundary migration, Phys. Rev. B 93 (2016) 024109. doi:10.1103/PhysRevB.93.024109

[9] N. Combe, F. Mompiou, M. Legros, Shear-coupled grain-boundary migration dependence on normal strain/stress, Phys. Rev. Materials 1 (2017) 033605. doi:10.1103/PhysRevMaterials.1.033605

[10] T. Gorkaya, D. A. Molodov, G. Gottstein, Stress-driven migration of symmetrical $\langle 100\rangle$ tilt grain boundaries in al bicrystals, Acta mat. 57 (2009) 5396-5405. doi:10.1016/j.actamat.2009.07.036

[11] D. A. Molodov, T. Gorkaya, G. Gottstein, Migration of the $\sigma 7$ tilt grain boundary in al under an applied external stress, Scripta Materialia 65 (11) (2011) 990 - 993. doi:10.1016/j.scriptamat.2011.08.030

[12] D. A. Molodov, T. Gorkaya, G. Gottstein, Dynamics of grain boundaries under applied mechanical stress, Journal Mater Sci. 46 (2011) 4318-4326. doi:10.1007/s10853-010-5233-6

[13] A. Rajabzadeh, F. Mompiou, M. Legros, N. Combe, Elementary mechanisms of shear-coupled grain boundary migration, Phys. Rev. Lett. 110 (2013) 265507. doi:10.1103/PhysRevLett.110.265507

[14] J. Han, S. L. Thomas, D. J. Srolovitz, Grain-boundary kinetics: A unified approach, Progress in Materials Science 98 (2018) 386 - 476. doi:10.1016/j.pmatsci.2018.05.004

[15] L. Wan, S. Wang, Shear response of the $\sigma 9\langle 110\rangle 221$ symmetric tilt grain boundary in fcc metals studied by atomistic simulation methods, Phys. Rev. B 82 (21) (2010) 214112. doi:10.1103/PhysRevB.82.214112

[16] H. Khater, A. Serra, R. Pond, J. Hirth, The disconnection mechanism of coupled migration and shear at grain boundaries, Acta Mat. 60 (2012) 2007-2020. doi:10.1016/j.actamat.2012.01.001

[17] A. Rajabzadeh, M. Legros, N. Combe, F. Mompiou, D. Molodov, Evidence of grain boundary dislocation step motion associated to shear-coupled grain boundary migration, Phil. Mag. 93 (2013) 1299. doi:10.1080/14786435.2012.760760

[18] A. Rajabzadeh, F. Mompiou, S. Lartigue-Korinek, N. Combe, M. Legros, D. Molodov, The role of disconnections in deformation-coupled grain boundary migration, Acta Mat. 77 (2014) 223-235. doi:10.1016/j.actamat.2014.05.062

[19] C. P. Race, J. von Pezold, J. Neugebauer, Role of the mesoscale in migration kinetics of flat grain boundaries, Phys. Rev. B 89 (2014) 214110. doi:10.1103/PhysRevB.89.214110

[20] Y. Deng, C. Deng, Size and rate dependent grain boundary motion mediated by disconnection nucleation, Acta Materialia 131 (2017) $400-409$. doi:10.1016/j.actamat.2017.04.018

[21] Q. Zhu, G. Cao, J. Wang, C. Deng, J. Li, Z. Zhang, S. X. Mao, In situ atomistic observation of disconnection-mediated grain boundary migration, Nature Comm. 10 (2019) 156. doi:10.1038/s41467-018-08031-x

[22] K. Chen, J. Han, S. L. Thomas, D. J. Srolovitz, Grain boundary shear coupling is not a grain boundary property, Acta Materialia 167 (2019) $241-247$. doi:10.1016/j.actamat.2019.01.040

[23] E. R. Homer, S. M. Foiles, E. A. Holm, D. L. Olmsted, Phenomenology of shear-coupled grain boundary motion in symmetric tilt and general grain boundaries, Acta Materialia 61 (4) (2013) 1048 - 1060. doi:10.1016/j.actamat.2012. 10.005

[24] K. Cheng, L. Zhang, C. Lu, K. Tieu, Coupled grain boundary motion in aluminium: the effect of structural multiplicity, Scientific Reports 6 (2016) 25427. doi:10.1038/srep25427.

[25] R. Gautier, A. Rajabzadeh, M. Larranaga, N. Combe, F. Mompiou, M. Legros, Shear-coupled migration of grain boundaries: the key missing link in the mechanical behavior of small-grained metals, Comptes Rendus Physique.

[26] L. Wan, S. Wang, Shear response of the $\sigma 11,\langle 110\rangle\{131\}$ symmetric tilt grain boundary studied by molecular dynamics, Modelling Simul. Matter. Sci. Eng. 17 (2009) 045008. doi:10.1088/0965-0393/17/4/045008

[27] L. Wan, J. Li, Shear responses of [110]-tilt $\{115\} /\{111\}$ asymmetric tilt grain boundaries in fcc metals by atomistic simulations, Modelling and Simulation in Materials Science and Engineering 21 (5) (2013) 055013. doi:10.1088/0965-0393/ $21 / 5 / 055013$

[28] Y. Deng, C. Deng, Atomic link between the structure and strength of grain boundaries subject to shear coupling, Phys. Rev. Materials 3 (2019) 010601. doi:10.1103/PhysRevMaterials.3.010601

[29] J. Hirth, R. Pond, Steps, dislocations and disconnections as interface defects relating to structure and phase transformations, Acta Mat. 44 (12) (1996) 4749 - 4763. doi:10.1016/S1359-6454(96)00132-2

[30] O. MacKain, M. Cottura, D. Rodney, E. Clouet, Atomic-scale modeling of twinning disconnections in zirconium, Phys. Rev. B 95 (2017) 134102. doi:10.1103/PhysRevB.95.134102

[31] A. Rajabazdeh, Experimental and theoretical study of the shear-coupled grain boundary migration, Ph.D. thesis, University of Toulouse (2013).

[32] E. R. Homer, S. Patala, J. L. Priedeman, Grain boundary plane orientation fundamental zones and structure-property relationships, Scientific reports 5 (2015) 15476. doi:10.1038/srep15476

[33] Y. Mishin, M. J. Mehl, D. A. Papaconstantopoulos, A. F. Voter, J. D. Kress, Structural stability and lattice defects in copper: Ab initio, tight-binding, and embedded-atom calculations, Phys. Rev. B 63 (2001) 224106. doi:10.1103/ PhysRevB.63.224106 
[34] G. Henkelman, B. P. Uberuaga, H. Jonsson, A climbing image nudged elastic band method for finding saddle points and minimum energy paths, J. Chem. Phys. 113 (22) (2000) 9901-9904. doi:10.1063/1.1329672

[35] S. J. Plimpton, Fast parallel algorithms for short-range molecular dynamics, J. Comp. Phys. 117 (1995) 1-19. doi: $10.1006 / \mathrm{jcph} .1995 .1039$

[36] S. J. Plimpton, R. Pollock, M. Stevens, Particle-mesh ewald and rrespa for parallel molecular dynamics simulations, in: Proc of the Eighth SIAM Conference on Parallel Processing for Scientific Computing, Minneapolis, 1997, p. 1.

[37] A. Serra, D. J. Bacon, A new model for 1012 twin growth in hcp metals, Philosophical Magazine A 73 (2) (1996) $333-343$. doi: $10.1080 / 01418619608244386$

[38] T. Frolov, D. L. Medlin, M. Asta, Dislocation content of grain boundary phase junctions and its relation to grain boundary excess properties, Phys. Rev. B 103 (2021) 184108. doi:10.1103/PhysRevB.103.184108

[39] L. Zhang, C. Lu, K. Tieu, X. Zhao, L. Pei, The shear response of copper bicrystals with $\sigma 11$ symmetric and asymmetric tilt grain boundaries by molecular dynamics simulation, Nanoscale 7 (2015) 7224. doi:10.1039/C4NR07496C

[40] F. Mompiou, M. Legros, D. Caillard, Smig model: A new geometrical model to quantify grain boundary-based plasticity, Acta Materialia 58 (10) (2010) 3676 - 3689. doi:10.1016/j.actamat.2010.03.003

[41] D. Caillard, F. Mompiou, M. Legros, Grain-boundary shear migration coupling. ii. geometrical model for general boundaries, Acta Mat. 57 (2009) 2390. doi:10.1016/j.actamat.2009.01.023

[42] D. E. Spearot, L. Capolungo, C. N. Tomé, Shear-driven motion of $\mathrm{mg} 10 \overline{1} 2$ twin boundaries via disconnection terrace nucleation, growth, and coalescence, Phys. Rev. Materials 3 (2019) 053606. doi:10.1103/PhysRevMaterials.3.053606

[43] D. Chen, S. Xu, Y. Kulkarni, Atomistic mechanism for vacancy-enhanced grain bouandary migration, Phys. Rev. Mat. 4 (2020) 033602. doi:10.1103/PhysRevMaterials.4.033602

[44] M. Larranaga, F. Mompiou, M. Legros, N. Combe, Role of sessile disconnection dipoles in shear-coupled grain boundary migration, Phys. Rev. Materials 4 (2020) 123606. doi:10.1103/PhysRevMaterials.4.123606

[45] S. L. Thomas, C. Wei, J. Han, Y. Xiang, D. J. Srolovitz, Disconnection description of triple-junction motion, Proceedings of the National Academy of Sciences 116 (18) (2019) 8756-8765. doi:10.1073/pnas.1820789116

[46] N. Combe, F. Mompiou, M. Legros, Heterogeneous disconnection nucleation mechanisms during grain boundary migration, Phys. Rev. Materials 3 (2019) 060601. doi:10.1103/PhysRevMaterials.3.060601.

[47] N. Kvashin, P. L. García-Müller, N. Anento, A. Serra, Atomic processes of shear-coupled migration in 112 twins and vicinal grain boundaries in bcc-fe, Phys. Rev. Materials 4 (2020) 073604. doi:10.1103/PhysRevMaterials.4.073604 